\title{
Sub-radian-accuracy gravitational waveforms of coalescing binary neutron stars in numerical relativity
}

\author{
Kenta Kiuchi, ${ }^{1}$ Kyohei Kawaguchi, ${ }^{2,1}$ Koutarou Kyutoku, ${ }^{3,1}$ \\ Yuichiro Sekiguchi, ${ }^{4}$ Masaru Shibata, ${ }^{1}$ and Keisuke Taniguchi ${ }^{5}$ \\ ${ }^{1}$ Center for Gravitational Physics, Yukawa Institute for Theoretical Physics, Kyoto University, Kyoto, 606-8502, Japan \\ ${ }^{2}$ Max Planck Institute for Gravitational Physics (Albert Einstein Institute), \\ Am Mhlenberg 1, Potsdam-Golm, 14476, Germany \\ ${ }^{3}$ Theory Center, Institute of Particle and Nuclear Studies, KEK, Tsukuba 305-0801, Japan \\ Department of Particle and Nuclear Physics, the Graduate University \\ for Advanced Studies (Sokendai), Tsukuba 305-0801, Japan \\ Interdisciplinary Theoretical Science (iTHES) Research Group, RIKEN, Wako, Saitama 351-0198, Japan \\ ${ }^{4}$ Department of Physics, Toho University, Funabashi, Chiba 274-8510, Japan \\ ${ }^{5}$ Department of Physics, University of the Ryukyus, Nishihara, Okinawa 903-0213, Japan
}

(Dated: August 31, 2017)

\begin{abstract}
Extending our previous studies, we perform high-resolution simulations of inspiraling binary neutron stars in numerical relativity. We thoroughly carry through a convergence study in our currently available computational resources with the smallest grid spacing of $\approx 63-86$ meter for the neutronstar radius $10.9-13.7 \mathrm{~km}$. The estimated total error in the gravitational-wave phase is of order $0.1 \mathrm{rad}$ for the total phase of $\gtrsim 210 \mathrm{rad}$ in the last $\sim 15-16$ inspiral orbits. We then compare the waveforms (without resolution extrapolation) with those calculated by the latest effective-one-body formalism (tidal SEOBv2 model referred to as TEOB model). We find that for any of our models of binary neutron stars, the waveforms calculated by the TEOB formalism agree with the numerical-relativity waveforms up to $\approx 3 \mathrm{~ms}$ before the peak of the gravitational-wave amplitude is reached: For this late inspiral stage, the total phase error is $\lesssim 0.1 \mathrm{rad}$. Although the gravitational waveforms have an inspiral-type feature for the last $\sim 3 \mathrm{~ms}$, this stage cannot be well reproduced by the current TEOB formalism, in particular, for neutron stars with large tidal deformability (i.e., lager radius). The reason for this is described.
\end{abstract}

PACS numbers: 04.25.D-, 04.30.-w, 04.40.Dg

\section{INTRODUCTION}

Gravitational-wave astronomy has vividly revealed its usefulness for exploring the nature of compact objects. Advanced LIGO operating since 2015 [1 has already reported three merger events of binary black holes [2 4], and the masses and spins of individual black holes have been successfully determined to modest accuracy despite the cosmological distances to these sources 5. A noteworthy feature of these events, particularly GW 150914 2], is that they are observed throughout the inspiral-merger-ringdown phases, which cannot be fully modeled by the traditional post-Newtonian $(\mathrm{PN})$ approximation suitable only for the early inspiral phase [6]. Thus, accurate theoretical waveforms applicable to dynamical phases are essential for the estimation of binary parameters [5] and also the test of general relativity 77. For this purpose, the effective-one-body (EOB) formalism calibrated by numerical-relativity simulations played a very important role in the data analysis (see Ref. [5] and references therein). As the quality of gravitational-wave data will be further improved in the near future with advanced Virgo 8 in operation since 2017 and upcoming KAGRA [9], accuracy of waveform models will become more important so as to avoid systematic errors.

The next target for ground-based detectors is gravita- tional waves from coalescing binary neutron stars (and also black hole-neutron star binaries), which will inform us about finite-size properties of neutron stars along with their masses. Simultaneous measurements of these quantities will become a powerful method to strongly constrain the not-yet-understood equation of state (EOS) of the neutron-star matter, and its accomplishment is one of the most important goals of gravitational-wave astronomy. On one hand, the masses of two neutron stars will be determined with high accuracy of $\lesssim 1 \%$ from gravitational-wave signals in the inspiral phase for a sufficiently high signal-to-noise ratio [10] as far as the neutron-star spins are small [11. On the other hand, it will be challenging to extract quantities associated with the finite-size effect, because it does not become appreciable until the very late inspiral phase.

Among various proposals, one of the most promising strategy is to read off tidal deformability, which governs the late-phase orbital evolution, from gravitational waves emitted during the inspiral phase up to the merger [12 18. This strategy requires an accurate template of gravitational waves from binary-neutron-star inspirals taking into account tidal deformation that influences the dynamics of the late inspiral orbits. In anticipation of coming detections of binary-neutron-star mergers, developing an accurate model of gravitational waveforms for binary-neutron-star inspirals including the tidal effects is an urgent task. 
The EOB formalism will play an important role also in the analysis of gravitational waves from binary neutron stars. Because the finite-size effect becomes important in the very late stage of the orbits, PN approximations with tidal effects [13] are not satisfactory. Indeed, it has been shown that the lack of knowledge about higher-order PN point-particle terms prevents us from accurately extracting the tidal deformability [17, 19, 23. The EOB formalism can improve the accuracy of the waveform templates for the dynamical phase via effective incorporation of higher-order PN terms and non-adiabatic dynamics close to merger. Motivated by this success, tidal EOB (TEOB) models have been developed by various authors to model the coalescence of binary neutron stars [15, 2428. These studies demonstrate that the extraction of tidal deformability is feasible for an event with a moderately high signal-to-noise ratio 28] (this fact is also confirmed by a numerical-relativity study [29]), or by stacking analysis of multiple events [18. All these results support the idea that extracting the tidal deformability from gravitational waves emitted in the late inspiral phase of binary-neutron-star mergers is a promising way to constrain the EOS of neutron-star matter.

However, Ref. 26] also suggests that the current TEOB approach is not yet accurate enough to model waveforms for the last few cycles for the case that the neutron-star radius is large. This implies that further modeling aided by high-precision waveforms derived by numerical relativity is required to obtain reliable templates in the final inspiral stage of neutron star binaries. For this purpose, a large-scale numerical-relativity simulation is crucial.

Long-term simulations for binary-neutron-star inspirals have recently been performed by several groups aiming at deriving high-precision numerical-relativity waveforms [21, 25, 29, 31, 41]. These work, in particular the latest ones, followed the late inspiral phase for $\gtrsim 10$ orbits up to the onset of merger. However, past numerical simulations would not be able to obtain gravitational waveforms with sufficient accuracy due to the following reasons. First in the early-stage work, initial data with an unphysical residual eccentricity were employed. This seriously degrades the accuracy of derived waveforms, because binary neutron stars in the late inspiral stage are believed to have a quasi-circular orbit with negligible eccentricity [42. This problem has been overcome, and simulations were performed with much less eccentric initial conditions in the latest work [21, 29, 31, 32, 41]. However, even in these recent work, the phase error in the waveforms was likely to be still of order $1 \mathrm{rad}$ because of the insufficient grid resolutions except for a single highest resolution model of Ref. 32.

In this paper, we push forward our previous numericalrelativity studies [21, 29] to a sub-100-meter-resolution regime. The simulations are performed for about 15-16 inspiral orbits employing the initial data in which the eccentricity is sufficiently small $\left(\sim 10^{-3}\right.$ : see appendix A) as in our previous studies 21, 29. The update lies in the grid resolution improved by a factor of up to $\sim 2.2$ from the previous ones. In the highest-resolution case, the minimum grid spacing is $63-86 \mathrm{~m}$ for the neutron stars of radius $10.9-13.7 \mathrm{~km}$ : The major diameter of neutron stars is covered by $\approx 270$ grid points. We show that the waveform depends very weakly on the grid spacing at such a high resolution, and the phase error in the gravitational waveforms is estimated to be of order $0.1 \mathrm{rad}$ among the total phase of $\gtrsim 210 \mathrm{rad}$. We then show that a TEOB model can be reliably calibrated with such highaccuracy numerical gravitational waveforms.

The paper is organized as follows. In Sec. II, we summarize the formulation and numerical schemes employed in our numerical-relativity study, and also review the adopted EOS. In Sec. III, we present numerical gravitational waveforms and show that the phase error in gravitational waves derived with our highest grid resolution is of order $0.1 \mathrm{rad}$. We then compare our best-resolved waveforms with those derived by the latest TEOB approach and examine the accuracy of the TEOB waveform in Sec. IV. Section V is devoted to a summary. Throughout this paper, we employ the geometrical units of $c=G=1$ where $c$ and $G$ are the speed of light and the gravitational constant, respectively.

\section{SUMMARY OF OUR SETTING FOR NUMERICAL-RELATIVITY SIMULATION}

In this section, we summarize the formulation and numerical schemes of our numerical-relativity simulations, EOS employed for neutron stars, definitions of the tidal deformability for binaries, and our recipe for constructing a waveform.

\section{A. Formulation, code, and models}

We follow the inspiral and early merger stages of binary neutron stars using a numerical-relativity code, SACRA [43]. Following our previous work [21, 29], we employ a moving puncture version of the BaumgarteShapiro-Shibata-Nakamura formalism [4], locally incorporating a Z4c-type constraint propagation prescription [45] (see Ref. [46] for our implementation) for a solution of Einstein's equation. In our numerical simulations, a fourth-order finite differencing scheme in space and time is used implementing an adaptive mesh refinement (AMR) algorithm (see Ref. 43] for details). In this work, we parallelized and tuned this AMR code significantly, and this improvement enables us to perform a number of high-resolution simulations in a relatively short time scale: As we describe later, the grid resolution is more than twice better (i.e., the grid spacing is by a factor $\lesssim 2$ smaller) than that in our previous work [21. The required CPU time is 540-650k core hours for the highest resolution models.

In this work, we prepare ten refinement levels for the AMR computational domain. Specifically, two sets of 
TABLE I. Model name, the location of outer boundaries along each axis denoted by $L$, and the finest grid spacing, $\Delta x_{\text {finest }}$, in several different grid-resolution runs. The model name reflects the EOS and mass of neutron stars. $\Delta x_{\text {finest }}$ is listed for $N=182,150,130,110,102$, and 90 in the equalmass models and $N=150,130,110,102$, and 90 in the unequal-mass models. We note that the wavelength of gravitational waves is initially $\lambda_{0} \approx 810 \mathrm{~km}$ irrespective of the models in this paper.

\begin{tabular}{lcclcl}
\hline \multicolumn{1}{c}{ Model } & $L(\mathrm{~km})$ & \multicolumn{3}{c}{$\Delta x_{\text {finest }}(\mathrm{m})$} \\
\hline B135-135 & 5860 & 63, & 76, & $88,104,112,127$ \\
HB135-135 & 6392 & 69,83, & $96,113,122,138$ \\
H135-135 & 6991 & 75,91, & $105,124,134,152$ \\
125H135-135 & 7324 & 79, & 95, & $110,130,140,159$ \\
15H135-135 & 7990 & $86,104,120,142,153,173$ \\
B121-151 & 5991 & 78, & $90,106,114,129$ \\
HB121-151 & 6324 & 82, & $95,112,121,137$ \\
H121-151 & 6823 & 89, & $103,121,131,148$ \\
125H121-151 & 7323 & 95, & $110,130,140,159$ \\
15H121-151 & 7822 & $102,118,138,150,170$ \\
\hline \hline
\end{tabular}

four finer domains comoving with each neutron star cover the region of their vicinity. The other six coarser domains cover both neutron stars by a wider domain with their origins fixed at the center of the mass of the binary system. Each refinement domain consists of a uniform, vertex-centered Cartesian grid with $(2 N+1,2 N+1, N+$ $1)$ grid points for $(x, y, z)$ (the equatorial plane symmetry at $z=0$ is imposed). The distance from the origin to outer boundaries along each axis is denoted by $L$. Here, $L$ is much larger than the initial wavelength of gravitational waves, $\lambda_{0}=\pi / \Omega_{0}$, with $\Omega_{0}$ being the initial orbital angular velocity (see Table I). We always choose it $\Omega_{0} \approx 0.0155 / m_{0}$ where $m_{0}$ is the total mass of the binary system at infinite separation.

In this work, we consider the models of total mass $m_{0} \approx 2.7 M_{\odot}$ (see Table II . More precisely, we select equal-mass models with each mass $m_{1}=m_{2}=1.35 M_{\odot}$ and unequal-mass models with each mass $m_{1} \approx 1.21 M_{\odot}$ and $m_{2} \approx 1.51 M_{\odot}$. For these models, the chirp mass defined by $\left(m_{1} m_{2}\right)^{3 / 5} m_{0}^{-1 / 5}$ (where $\left.m_{0}=m_{1}+m_{2}\right)$ is fixed to be $\approx 1.17524 M_{\odot}$. For the unequal-mass models, the symmetric mass ratio defined by $\eta:=m_{1} m_{2} / m_{0}^{2}$ is chosen to be 0.247 (i.e., the corresponding mass ratio $q=m_{1} / m_{2}$ is chosen to be $\approx 0.8025$ ). For these values of $m_{0}, \lambda_{0} \approx 810 \mathrm{~km}$ and initial gravitational-wave frequency $f \approx 370 \mathrm{~Hz}$.

The grid spacing for each domain is $\Delta x_{l}=L /\left(2^{l} N\right)$, where $l=0-9$. In this work, we choose a wide variety of values for $N$ and examine the convergence properties of numerical results: For the equal-mass models, we perform the simulations with $N=182,150,130,110$, 102 , and 90 and for the unequal-mass models, $N=150$, $130,110,102$, and 90 . We note that in our previous work [21, 29], $N$ was at best 72 [30. With the high-
TABLE II. Equations of state employed, the radius, $R_{M}$, and the dimensionless tidal deformability $\Lambda_{M}$ of spherical neutron stars of $M=1.21,1.35$, and $1.51 M_{\odot} . R_{M}$ is listed in units of $\mathrm{km}$.

\begin{tabular}{ccccccc}
\hline \hline EOS & $R_{1.21}$ & $R_{1.35}$ & $R_{1.51}$ & $\Lambda_{1.21}$ & $\Lambda_{1.35}$ & $\Lambda_{1.51}$ \\
\hline B & 10.98 & 10.96 & 10.89 & 581 & 289 & 131 \\
HB & 11.60 & 11.61 & 11.57 & 827 & 422 & 200 \\
H & 12.25 & 12.27 & 12.26 & 1163 & 607 & 298 \\
$125 \mathrm{H}$ & 12.93 & 12.97 & 12.98 & 1621 & 863 & 435 \\
$15 \mathrm{H}$ & 13.63 & 13.69 & 13.73 & 2238 & 1211 & 625 \\
\hline \hline
\end{tabular}

est grid resolution, $N=182$, the semi-major diameter of each neutron star is covered by about 270 grid points. For the simulation with a small-radius neutron star of radius $10.9 \mathrm{~km}$, the best grid spacing is $\approx 63 \mathrm{~m}$ (see Table $\mathrm{I}$ ). With our setting of $m_{0} \Omega_{0} \approx 0.0155$, the binary experiences $15-16$ orbits before the gravitational-wave amplitude reaches a peak.

We prepare binary neutron stars in quasi-circular orbits with small eccentricity $\sim 10^{-3}$ for the initial condition of numerical simulations. These initial conditions are numerically obtained by using a spectral-method library, LORENE [47. The eccentricity reduction is performed by the method of Ref. [46. The neutron stars are assumed to have an irrotational velocity field, which is believed to be an astrophysically realistic (or at least approximately realistic) configuration [48, 49].

We note that even with the eccentricity-reduced initial conditions, the small residual eccentricity of $\sim 10^{-3}$ still gives a small damage for getting accurate quasi-circular waveform. This is in particular the case for carefully comparing the numerical waveforms with those by TEOB formalisms (see the discussion in Appendix A). The numerical waveforms for the first 3-4 orbits are not very suitable for performing the careful analysis of gravitational-wave data. Thus, when comparing the numerical waveforms with those by the TEOB formalisms, we only employ the waveforms for the last $11-12$ orbits. We first notice this fact when we obtain the results of high-resolution simulations in this paper. This finding reconfirms that the eccentricity reduction for constructing the initial data is crucial for accurately deriving the late inspiral waveforms.

\section{B. Equations of State}

Following our previous work [16, 34, 51, we employ a parameterized piecewise-polytropic EOS [50] with two pieces. In this work, our purpose is to accurately clarify the dependence of inspiral gravitational waveforms on the tidal deformability. For this purpose, the choice of the simple EOS is acceptable.

This EOS is written in terms of two segments of poly- 
tropes of the form

$$
P= \begin{cases}K_{0} \rho^{\Gamma_{0}} & \left(\text { for } \rho_{0} \leq \rho<\rho_{1}\right) \\ K_{1} \rho^{\Gamma_{1}} & \left(\text { for } \rho_{1} \leq \rho\right)\end{cases}
$$

where $\rho$ is the rest-mass density, $P$ is the pressure, $K_{0}$ and $K_{1}$ are a polytropic constant, and $\Gamma_{0}$ and $\Gamma_{1}$ are an adiabatic index. At the boundary of these two piecewise polytropes, $\rho=\rho_{1}$, the pressure is required to be continuous, i.e., $K_{0} \rho_{1}^{\Gamma_{0}}=K_{1} \rho_{1}^{\Gamma_{1}}$. Thus, the parameters, which have to be given, are $K_{0}, \rho_{1}, \Gamma_{0}$, and $\Gamma_{1}$. Following the previous studies [16, 34, 51, these parameters are determined in the following manner: The low-density EOS is fixed by setting $\Gamma_{0}=1.3562395$ and $K_{0}=3.594 \times 10^{13}$ in cgs units. The adiabatic index for the high-density region is set to be $\Gamma_{1}=3$, and hence, $K_{1}$ is determined to be $K_{1}=K_{0} \rho_{1}^{\Gamma_{0}-\Gamma_{1}}$. The remaining parameter, $\rho_{1}$, is varied for a wide range to prepare neutron stars with a variety of the radius and tidal deformability (see Table II). We note that for any EOS employed in this paper, the maximum mass of spherical neutron stars is larger than $2.0 M_{\odot}$; the approximate maximum mass for neutron stars for which the mass is accurately measured to date [52] (see Ref. [51] on the data of the maximum mass for each EOS).

In numerical simulations, we employ the following modified version of the piecewise polytropic EOS to approximately take into account thermal effects:

$$
\begin{aligned}
P & =P_{\text {cold }}(\rho)+\left(\Gamma_{\mathrm{th}}-1\right) \rho \varepsilon_{\mathrm{th}}, \\
\varepsilon & =\varepsilon_{\text {cold }}(\rho)+\varepsilon_{\mathrm{th}},
\end{aligned}
$$

where $\Gamma_{\text {th }}$ is a constant. The cold parts (the first terms) of both variables are calculated using the original piecewise polytropic EOS from $\rho$, and then the thermal part of the specific internal energy is determined from $\varepsilon$ as $\varepsilon_{\text {th }}=\varepsilon-\varepsilon_{\text {cold }}(\rho)$. Because $\varepsilon_{\text {th }}$ vanishes in the absence of shock heating, it is regarded as the finite-temperature part determined by the shock heating in the present context. Following our latest work [21, 29, 46, $\Gamma_{\text {th }}$ is chosen to be 1.8 , but this is not relevant for the present study because we focus only on the late inspiral evolution.

\section{Parameters associated with tidal deformability}

For modeling the late inspiral orbital motion and corresponding gravitational waves of binary neutron stars, two parameters constructed from the dimensionless tidal deformability of two neutron stars are often used. One is the EOB tidal parameter [28] which appears in the equation of motion of the TEOB formalism and is defined by

$$
\Lambda_{T}:=16 \eta\left(X_{1}^{3} \Lambda_{1}+X_{2}^{3} \Lambda_{2}\right)
$$

where $X_{i}:=m_{i} / m_{0}$ and $\Lambda_{i}$ is the dimensionless tidal deformability of each neutron star. We note that the originally defined variable is $\kappa_{2}^{T}$ and it is calculated by $3 \Lambda_{T} / 16$.
The other parameter is the so-called binary tidal deformability, $\tilde{\Lambda}$, defined by [17]

$$
\begin{aligned}
\tilde{\Lambda}=\frac{8}{13}[ & \left(1+7 \eta-31 \eta^{2}\right)\left(\Lambda_{1}+\Lambda_{2}\right) \\
& \left.-\sqrt{1-4 \eta}\left(1+9 \eta-11 \eta^{2}\right)\left(\Lambda_{1}-\Lambda_{2}\right)\right] .
\end{aligned}
$$

where we supposed that $m_{1} \leq m_{2}$ and then, $\Lambda_{1} \geq \Lambda_{2}$. $\tilde{\Lambda}$ is related to the leading term associated with the tidal effect in the gravitational-wave phase in the Fourier space, and hence, in the real gravitational-wave detection, this can be regarded as the primarily measured quantity.

One interesting property for $\Lambda_{T}$ and $\tilde{\Lambda}$ is that for a fixed chirp mass, these values are in a narrow range (within 1\% disagreement) as long as we consider the cases that $m_{0} \approx 2.7 M_{\odot}$ and $0.8 \leq q \leq 1$. Hence, in the following, we refer only to $\tilde{\Lambda}$, supposing that $\tilde{\Lambda}$ agrees approximately with $\Lambda_{T}$. Note that for the equal-mass models $(\eta=0.25), \Lambda_{T}=\tilde{\Lambda}$.

\section{Extraction of gravitational waves}

As a first step toward producing gravitational waves from numerical data, we extract the outgoing-component of complex Weyl scalar $\Psi_{4}$ [4]. If $\Psi_{4}$ is extracted at a sufficiently large radius, complex gravitational waveforms are determined in spherical coordinates $(r, \theta, \phi)$ by

$$
h(t, \theta, \varphi)=-\lim _{r \rightarrow \infty} \int^{t} d t^{\prime} \int^{t^{\prime}} d t^{\prime \prime} \Psi_{4}\left(t^{\prime \prime}, r, \theta, \varphi\right) .
$$

$\Psi_{4}$ can be expanded with respect to the spin-weighted spherical harmonics of weight $-2,{ }_{-2} Y_{l m}$, as

$$
\Psi_{4}(t, r, \theta, \phi)=\sum_{l m} \Psi_{4}^{l, m}(t, r)_{-2} Y_{l m}(\theta, \phi),
$$

where $\Psi_{4}^{l, m}$ is the expansion coefficient defined by this equation. In this work, we focus only on the $(l,|m|)=$ $(2,2)$ mode because we pay attention only to the equalmass or nearly equal-mass binary, and hence, this quadrupole mode is the dominant one.

We extract $\Psi_{4}$ at a finite spherical-coordinate radius of $r \approx 200 m_{0}$, and then, calculate $\Psi_{4}^{2,2}$ as a function of the retarded time defined by

$$
t_{\text {ret }}:=t-r_{*},
$$

where $r_{*}$ is the so-called tortoise coordinate defined by

$$
r_{*}:=r_{\mathrm{A}}+2 m_{0} \ln \left(\frac{r_{\mathrm{A}}}{2 m_{0}}-1\right),
$$

with $r_{\mathrm{A}}:=\sqrt{A / 4 \pi}$ and $A$ the proper area of the extraction sphere.

Since $\Psi_{4}^{2,2}\left(t_{\text {ret }}\right)$ extracted at a finite radius, $r_{0} \approx$ $200 m_{0}$, is different from the true gravitational waveform observed at null infinity, we then have to compute an 

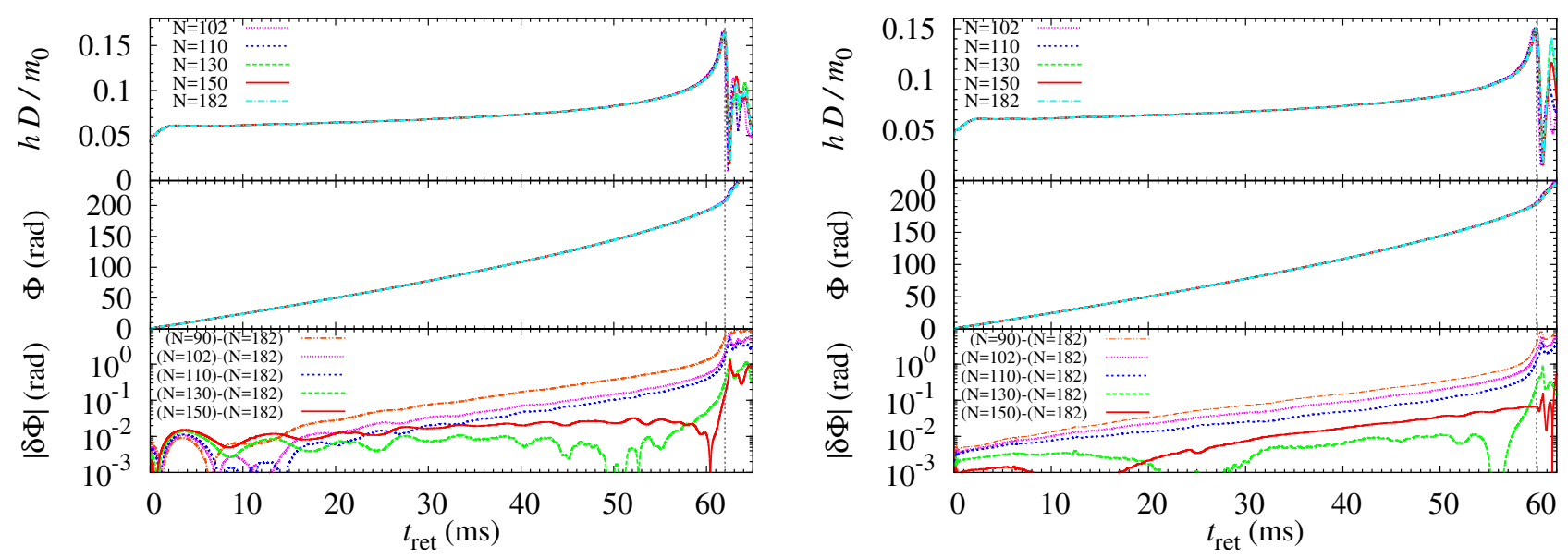

FIG. 1. The amplitude $\left(A^{2,2} D / m_{0}\right.$; upper panels) and phase ( $\Phi$; middle panels) of numerical gravitational waveforms with different grid resolutions for the equal-mass models with HB EOS (left) and $125 \mathrm{H}$ EOS (right). In the bottom panels, we also plot the difference in phase with respect to the best grid resolution $(N=182$ case) for $N=90,102,110,130$, and 150 . The vertical lines denote the time at which the peak amplitude is reached for $N=182$. $D$ denotes the distance to the source.

extrapolated waveform at $r_{0} \rightarrow \infty$. As in our previous studies [21, 29], for this purpose, we employ the Nakano's method (approximately equivalent to the Cauchy matching method) [53 55], by which the waveform at infinity is calculated by

$$
\begin{aligned}
\Psi_{4}^{l, m, \infty}\left(t_{\mathrm{ret}}, r_{0}\right)= & C\left(r_{0}\right)\left[\Psi_{4}^{l, m}\left(t_{\mathrm{ret}}, r_{0}\right)\right. \\
& \left.-\frac{(l-1)(l+2)}{2 r_{\mathrm{A}}} \int^{t_{\mathrm{ret}}} \Psi_{4}^{l, m}\left(t^{\prime}, r_{0}\right) d t^{\prime}\right],
\end{aligned}
$$

where $C\left(r_{0}\right)$ is a function of $r_{0}$. Since our coordinates are similar to isotropic coordinates of non-rotating black holes, we choose $r_{\mathrm{A}}=r_{0}\left[1+m_{0} /\left(2 r_{0}\right)\right]^{2}$ and $C\left(r_{0}\right)=$ $1-2 m_{0} / r_{\mathrm{A}}$. In this setting, $t_{\text {ret }}$ at $r=r_{0}$ is given by Eqs. (2.8) and (2.9).

We also perform the same analysis choosing different extraction radii as $r_{0} / m_{0}=156$ and 178 and estimate the error of the gravitational-wave phase coming from the extraction of $\Psi_{4}$ at finite radii, because the effect of the finite-radius extraction still remains in $\Psi_{4}^{l, m, \infty}$.

For $\Psi_{4}^{l, m, \infty}\left(t_{\text {ret }}, r_{0}\right)$ thus determined, the gravitational waveform of each mode is obtained by twice integrating it as [see Eq. 2.6] ]

$$
\begin{aligned}
h^{l, m}\left(t_{\mathrm{ret}}\right) & :=h_{+}^{l, m}\left(t_{\mathrm{ret}}\right)-i h_{\times}^{l, m}\left(t_{\mathrm{ret}}\right) \\
& =-\int_{t_{\mathrm{ret}}} d t^{\prime} \int_{t^{\prime}} d t^{\prime \prime} \Psi_{4}^{l, m, \infty}\left(t^{\prime \prime}\right) .
\end{aligned}
$$

For this integration, we employ the method of Ref. [56], and write $h^{l, m}\left(t_{\text {ret }}\right)$ as

$$
h^{l, m}\left(t_{\mathrm{ret}}\right)=\int d \omega^{\prime} \frac{\Psi_{4}^{l, m, \infty}\left(\omega^{\prime}\right)}{\max \left(\omega^{\prime}, \omega_{\mathrm{cut}}\right)^{2}} \exp \left(i \omega^{\prime} t_{\mathrm{ret}}\right),
$$

where $\Psi_{4}^{l, m, \infty}(\omega)$ is the Fourier transform of $\Psi_{4}^{l, m, \infty}\left(t_{\text {ret }}\right)$ and $\omega_{\text {cut }}$ is chosen to be $1.6 \Omega_{0}$. (Note that at the initial stage, the value of $\omega$ is $\left.\approx 2 \Omega_{0}>\omega_{\text {cut }}\right)$. We recall again that in this paper we pay attention only to $l=|m|=2$ modes because these are the dominant modes in particular for the equal-mass binaries.

From Eq. 2.12), the evolution of the amplitude, i.e., $A^{l, m}=\left|h^{l, m}\right|$, is immediately determined. For the analysis employed in our method, we can also define the angular frequency

$$
\omega\left(t_{\mathrm{ret}}\right):=\frac{\left|\dot{h}^{2,2}\right|}{\left|h^{2,2}\right|}
$$

and subsequently, the gravitational-wave phase by

$$
\Phi\left(t_{\mathrm{ret}}\right):=\int^{t_{\mathrm{ret}}} d t^{\prime} \omega\left(t^{\prime}\right)
$$

Now, using $A^{2,2}$ and $\Phi$, the quadrupole gravitational waveform can be written as

$$
h^{2,2}\left(t_{\mathrm{ret}}\right)=A^{2,2}\left(t_{\mathrm{ret}}\right) \exp \left[i \Phi\left(t_{\mathrm{ret}}\right)\right] .
$$

Before closing this section, we note that the angular frequency defined by Eq. (2.13) is contaminated by the time derivative of the amplitude. Although the associated error for our current analysis that focuses only on the inspiral phase is negligible, future analysis of more dynamical merger phase will require more appropriate definitions such as [57]

$$
\omega_{\mathrm{dyn}}\left(t_{\mathrm{ret}}\right):=\operatorname{Im}\left(\frac{h^{* 2,2} \dot{h}^{2,2}}{\left|h^{2,2}\right|^{2}}\right),
$$

where $h^{* 2,2}$ is the complex conjugate of $h^{2,2}$. 

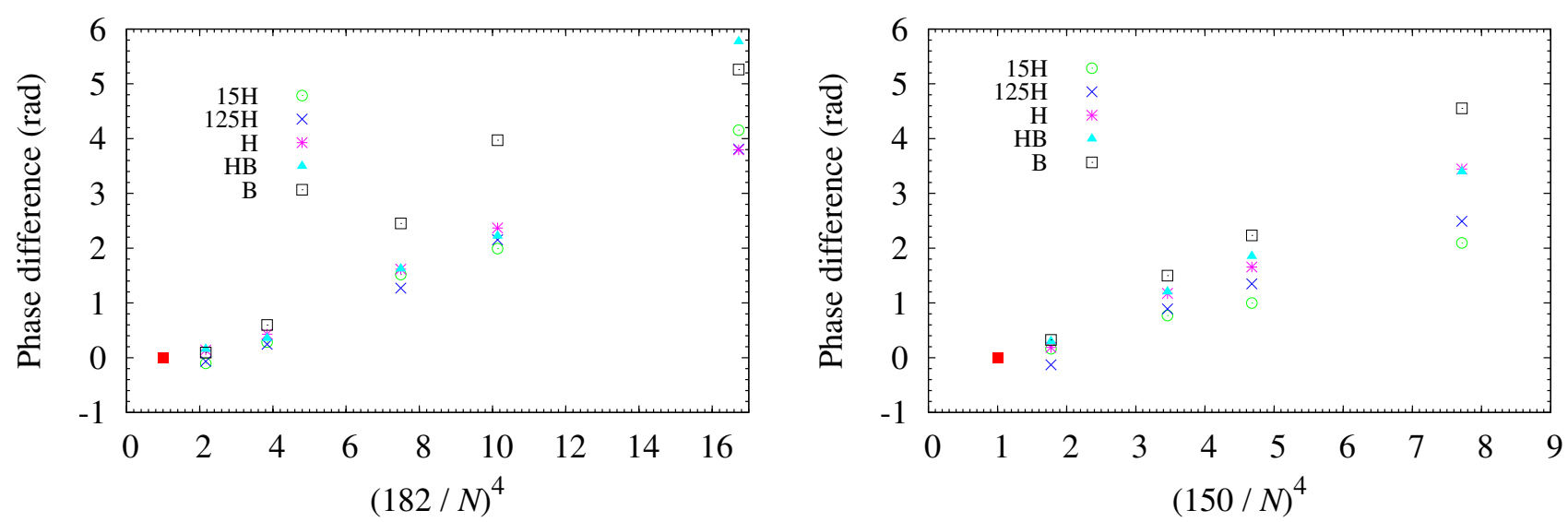

FIG. 2. Difference in the gravitational-wave phase, $\Phi(t)$, between the results of the best-grid resolution and others at the moment that the gravitational-wave amplitude for the best-grid resolution reaches the peak as a function of $(182 / N)^{4}$ for the equal-mass models (left) and as a function of $(150 / N)^{4}$ for the unequal-mass models (right) with 5 different EOS models.
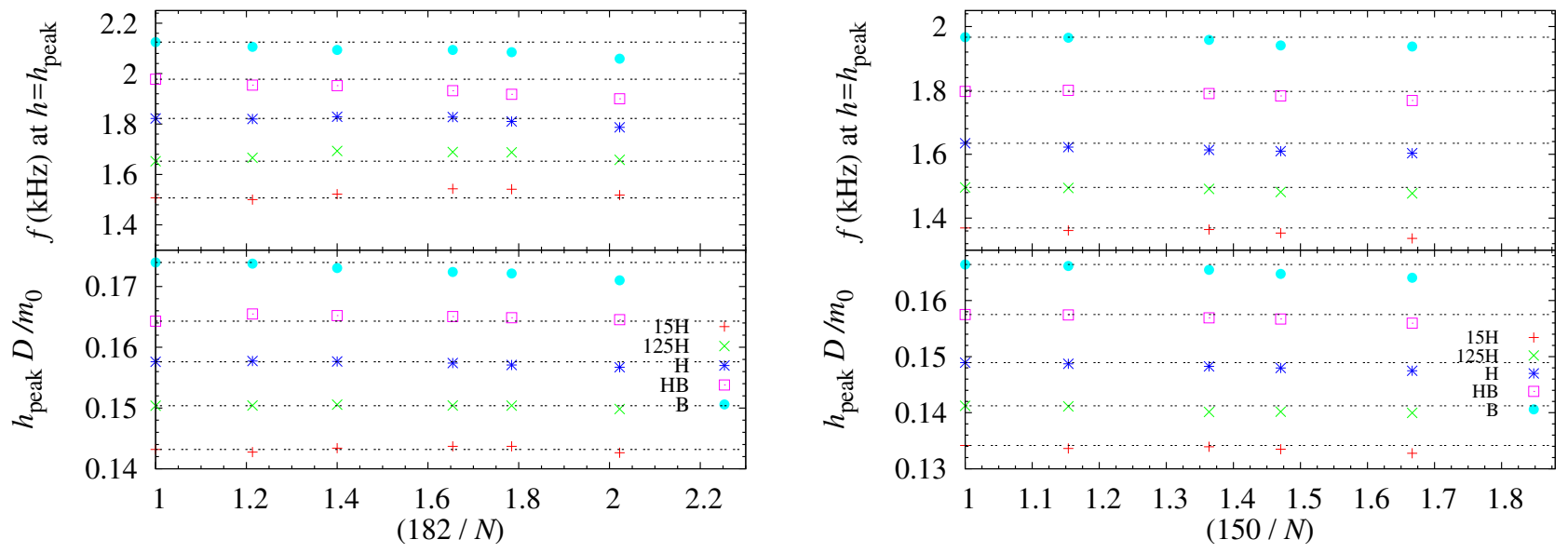

FIG. 3. The frequency of gravitational waves at the peak (top panel) and peak amplitude of gravitational waves (bottom panel) as functions of $182 / N$ for the equal-mass models (left panel) and as functions of $150 / N$ for the unequal-mass models (right panel). The dot-dot horizontal lines denote the values of the best grid resolution models.

\section{NUMERICAL RESULTS}

Figure 1 plots the amplitude $\left(A^{2,2} D / m_{0}\right.$; upper panels) and phase ( $\Phi$; middle panels) of numerical gravitational waveforms with different grid resolutions for the equal-mass models with HB EOS (left) and $125 \mathrm{H}$ EOS (right). $D$ denotes the distance to the source. In the bottom panels for both left and right, we also plot the difference in phase with respect to the best grid-resolution $(N=182)$ results for $N=90,102,110,130$, and 150 . This figure shows that the merger occurs earlier for the poor grid resolutions with $N \lesssim 130$. Specifically, the evolution of $\Phi\left(t_{\text {ret }}\right)$ is spuriously accelerated for such low grid resolutions. However, for the high grid resolutions with $N \gtrsim 150$, the phase evolution depends only weakly on the grid resolution. In some models like $15 \mathrm{H}$ and $125 \mathrm{H}$ models, the merger for $N=182$ occurs slightly earlier than for $N=150$. However, the peak amplitude time difference is as small as $\approx 0.5$ microsecond. When we pay attention to the waveforms only up to the peak amplitude, the phase difference between $N=150$ and $N=182$ is $0.1-0.2 \mathrm{rad}$ irrespective of the models (see Fig. 22.

The left panel of Fig. 2 plots differences in the gravitational-wave phase, $\Phi\left(t_{\text {ret }}\right)$, at the moment that the gravitational-wave amplitude for $N=182$ reaches the peak as a function of $(182 / N)^{4}$ for the equal-mass models with five different EOS. This shows that the phase difference steeply (as fast as or faster than the fourth-order convergence) decreases with the improvement of the grid resolution for $N \gtrsim 100$ irrespective of the EOS. In particular for $N \gtrsim 150$, the phase difference is decreased to 0.1-0.2 rad, and the phase error appears to be convergent (besides an irregular error that does not converge monotonically with the improvement of the grid resolution: see below for a discussion). This indicates that for 


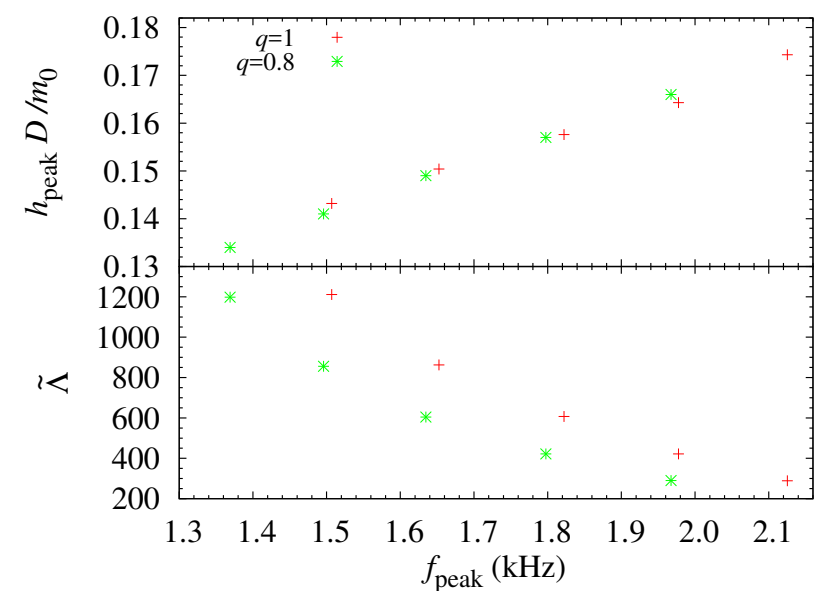

FIG. 4. Relation between the peak amplitude and the corresponding frequency of gravitational waves (top panel), and between the value of $\tilde{\Lambda}$ and the peak frequency (bottom panel). We show the result of $N=182$ for the equal-mass models and $N=150$ for the unequal-mass models.

$N \gtrsim 150\left(\Delta x_{9} \lesssim 100 \mathrm{~m}\right)$, nearly convergent waveforms with the phase error within $\sim 0.2$ rad would be obtained.

The right panel of Fig. 2 plots differences in the gravitational-wave phase for the unequal-mass models. It is found that the convergence behavior of the phase difference is similar to that for the equal-mass models. This suggests that for $N=150$, a nearly convergent waveform is likely to be also obtained for these unequal-mass models.

Figure 1 shows that for $N \lesssim 110$, the phase difference monotonically decreases with the improvement of the grid resolution. However, for $N \gtrsim 130$, the phase difference does not show such monotonic behavior as already mentioned. This indicates the presence of an unidentified source of the numerical error that does not monotonically converge with the improvement of the grid resolution. Figure 1 indicates that such error source generates the error in gravitational-wave phase by $\sim 0.1 \mathrm{rad}$ irrespective of the EOS employed.

The another phase error sources are the finite-radius extraction and the violation of the baryon mass conservation. In Appendix B, we show the phase error due to the finite-radius extraction is less than $0.04 \mathrm{rad}$ irrespective of the models.

The violation of the baryon mass conservation may cause a phase error 31]. We also show that this error is much smaller than $0.01 \mathrm{rad}$ up to the merger irrespective of the models in the appendix. Therefore, we have to bear in mind that in our current numerical waveform, the phase error of $\sim 0.1 \mathrm{rad}$ cannot be avoided.

Figure 3 plots the peak amplitude of gravitational waves, $h_{\text {peak }}$, and the frequency at the peak, $f_{\text {peak }}$, as functions of the grid resolution, described by $(182 / N)$, for the equal-mass models (left panel) and as functions of $(150 / N)$ for the unequal-mass models (right panel).
This figure shows that the quantities associated with the peak depend weakly on the grid resolution. In particular, it is found that $f_{\text {peak }}$ may be underestimated if the grid resolution is not high enough. It should be also remarked that the fluctuation in $f_{\text {peak }}$ is rather large even among the high-resolution results. This is reasonable because the frequency rapidly increases near the amplitude peak. We should keep in mind that the value of the peak frequency has an error of $2-3 \%$.

Figure 4 plots the relation between $h_{\text {peak }}$ and $f_{\text {peak }}$ and between $\tilde{\Lambda}$ and $f_{\text {peak }}$ following Ref. [16. This shows that the relation between $h_{\text {peak }}$ and $f_{\text {peak }}$ depends only weakly on the mass ratio. Qualitatively this is reasonable because $h_{\text {peak }}$ should be an increasing function of $f_{\text {peak }}$. It is also found that the relation between $\tilde{\Lambda}$ and $f_{\text {peak }}$ depends strongly on the mass ratio. We note that $\tilde{\Lambda}$ is approximately equal to $\Lambda_{T}$ for the models employed in this paper. Thus, our results do not show a universal relation (see also Ref. [58]). Nevertheless, Fig. 4 shows that $f_{\text {peak }}$ (and hence $h_{\text {peak }}$ ) has valuable information on $\tilde{\Lambda}$ as follows: (i) if $f_{\text {peak }}$ is higher than $\sim 2 \mathrm{kHz}$ (i.e., $\left.h_{\text {peak }} D / m_{0} \gtrsim 0.16\right)$ for the chirp mass of $\approx 1.1752 M_{\odot}$, $\tilde{\Lambda} \lesssim 500$, implying that the EOS is rather soft. (ii) if $f_{\text {peak }}$ is lower than $\sim 1.4 \mathrm{kHz}$ (i.e., $h_{\text {peak }} D / m_{0} \lesssim 0.14$ ) for the chirp mass of $\approx 1.1752 M_{\odot}, \tilde{\Lambda} \gtrsim 1000$, implying that the EOS is rather stiff.

\section{COMPARISON BETWEEN NUMERICAL-RELATIVITY AND TEOB WAVEFORMS}

In this section, we compare the NR waveforms with those by a TEOB formalism. For the TEOB formalism, we employ a latest version reported in Ref. [26], in which the effects of not only the static but also dynamical tides are taken into account.

In this comparison, we use the NR waveforms without performing extrapolation to the limit of $\Delta x_{\text {finest }}=0$ because as we showed in the previous section (and found in this section), the numerical error of the waveforms is small enough to perform the direct comparison. In the following, we employ the NR waveforms obtained for $N=182$ or $N=150$ for the comparison: For the equalmass models, we use the two waveforms of different grid resolutions and show that the results on the comparison do not lead to any serious difference.

When comparing two waveforms, we first have to align the time and phase of the NR and TEOB waveforms. This is done by searching for the minimum of the following correlation, $I_{c}$, varying $\tau$ and $\phi$ :

$$
\begin{aligned}
I_{c}= & \min _{\tau, \phi} \int_{t_{i}}^{t_{f}} d t_{\mathrm{ret}} \mid A_{\mathrm{NR}}^{2,2}\left(t_{\mathrm{ret}}\right) \exp \left[i \Phi_{\mathrm{NR}}\left(t_{\mathrm{ret}}\right)\right] \\
& -\left.A_{\mathrm{TEOB}}^{2,2}\left(t_{\mathrm{ret}}+\tau\right) \exp \left[i \Phi_{\mathrm{TEOB}}\left(t_{\mathrm{ret}}+\tau\right)+i \phi\right]\right|^{2} .
\end{aligned}
$$



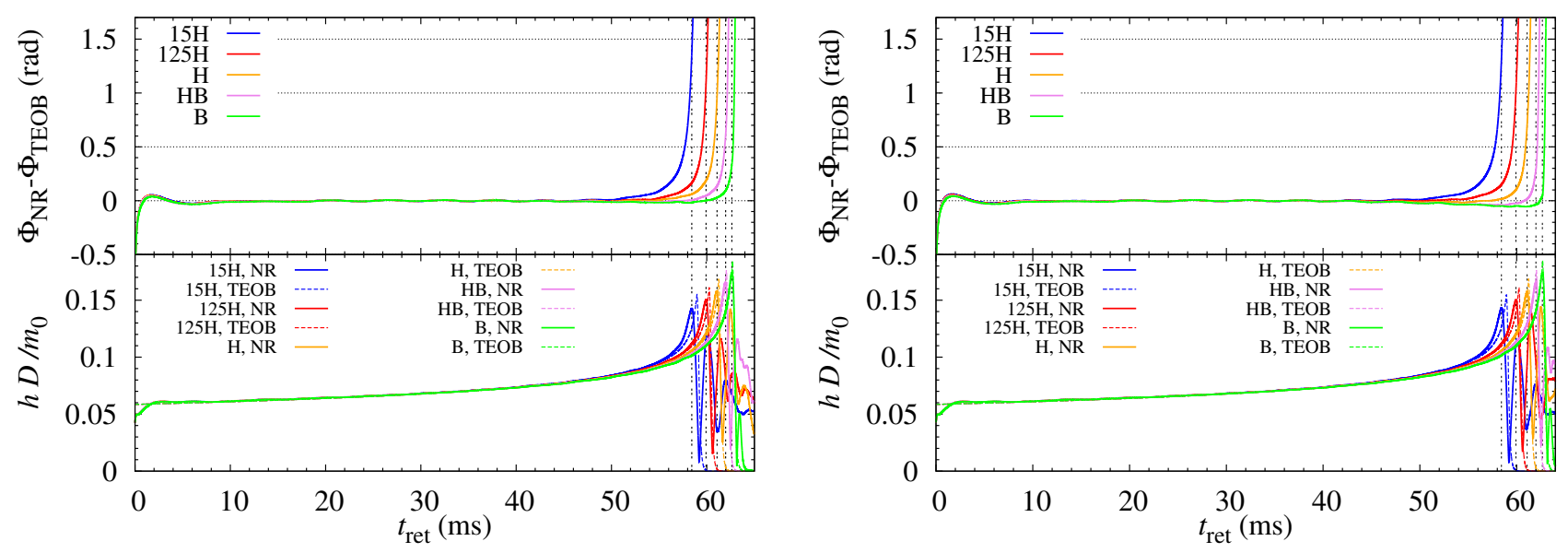

FIG. 5. Comparison between NR and TEOB waveforms for the equal-mass models. For the NR waveforms, we use the data with $N=150$ (left) and 182 (right). The top and bottom panels show the phase difference, $\Phi_{\mathrm{NR}}-\Phi_{\mathrm{TEOB}}$, and amplitude, $A^{2,2} D / m_{0}$ of gravitational waves, respectively. Here, $D$ denotes the distance to the source. The vertical dashed lines are the peak amplitude time $t_{\text {peak }}$ when the gravitational-wave amplitude reaches a peak in each model.

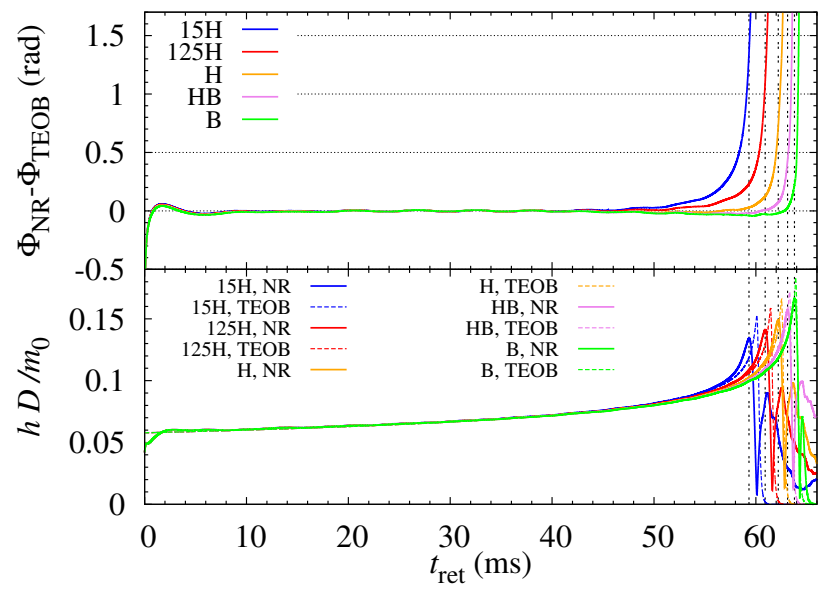

FIG. 6. The same as Fig. 5 but for the unequal-mass models. For the NR waveforms, we use the data with $N=150$.

Here, $A_{\mathrm{NR}}^{2,2}$ and $\Phi_{\mathrm{NR}}$ denote the amplitude and phase of gravitational waves for the numerical-relativity data, respectively. $A_{\mathrm{TEOB}}^{2,2}$ and $\Phi_{\mathrm{TEOB}}$ denote those by the TEOB formalism. For calculating the correlation, we employ the time domain NR waveforms of $20 \mathrm{~ms} \leq t_{\text {ret }} \leq$ $40 \mathrm{~ms}$. The corresponding gravitational-wave frequency at $t_{\mathrm{ret}}=20$ and $40 \mathrm{~ms}$ is $\approx 410$ and $500 \mathrm{~Hz}$, respectively.

The reason that we choose the rather late-time NR waveforms of $20 \mathrm{~ms} \leq t_{\text {ret }} \leq 40 \mathrm{~ms}$ for the correlation $I_{c}$ is as follows: In the early stage of the numerical evolution with $t_{\text {ret }} \lesssim 15 \mathrm{~ms}$, the frequency of gravitational waves always has an irregular modulation (see Appendix A). For precisely comparing the NR waveforms with those by the TEOB approach, such modulation, even if its amplitude is not very large, introduces the uncertainty in matching. To remove such uncertainty, we discard the waveforms in
TABLE III. $\Phi_{\mathrm{NR}}-\Phi_{\mathrm{TEOB}}$ at $t=t_{\mathrm{peak}}$ in units of radian for the equal-mass models with $N=182$ and 150, and for the unequal-mass models with $N=150$.

\begin{tabular}{cccc}
\hline \hline EOS & $(\eta, N)=(0.250,182)$ & $(0.250,150)$ & $(0.247,150)$ \\
\hline $\mathrm{B}$ & 0.1 & 0.3 & 0.2 \\
$\mathrm{HB}$ & 0.3 & 0.6 & 0.4 \\
$\mathrm{H}$ & 0.7 & 0.9 & 0.7 \\
$125 \mathrm{H}$ & 1.0 & 1.1 & 1.1 \\
$15 \mathrm{H}$ & 1.3 & 1.3 & 1.3 \\
\hline \hline
\end{tabular}

the early stage. We note that even for $t_{\text {ret }} \geq 20 \mathrm{~ms}$, there are $\gtrsim 22$ wave cycles ( $\gtrsim 11$ orbits) in our numerical data. We confirmed that the choice of the time-window of the matching does not significantly affect the following result.

In Figs. 5 and 6, we compare the NR waveforms with the TEOB waveforms. In Table [II] we also list $\Phi_{\mathrm{NR}}-\Phi_{\mathrm{TEOB}}$ at the moment that the gravitational-wave amplitude reaches the peak (referred to as $t_{\text {peak }}$ in the following). Figures 5 and 6 show that up to $t_{\text {peak }}-3 \mathrm{~ms}$, the TEOB waveforms well reproduce the NR waveforms irrespective of the EOS and mass ratio: In particular for the models for which the compactness is large and the tidal deformability is small (e.g., for B EOS), the agreement is quite good even at $t=t_{\text {peak }}-1 \mathrm{~ms}$, and the disagreement in the gravitational-wave phase is within $0.3 \mathrm{rad}$ up to $t_{\text {peak }}$, which is within the uncertainty due to the phase error.

On the other hand, for the models for which the compactness is relatively small (e.g., for $125 \mathrm{H}$ and $15 \mathrm{H} \mathrm{EOS}$ ), agreement between the NR and TEOB waveforms becomes poor for the last few ms prior to $t_{\text {peak }}$, leading to a phase disagreement of $\gtrsim 1 \mathrm{rad}$, which is greater than the uncertainty due to the phase error (see Table III). The interpretation for this is described as follows: For 

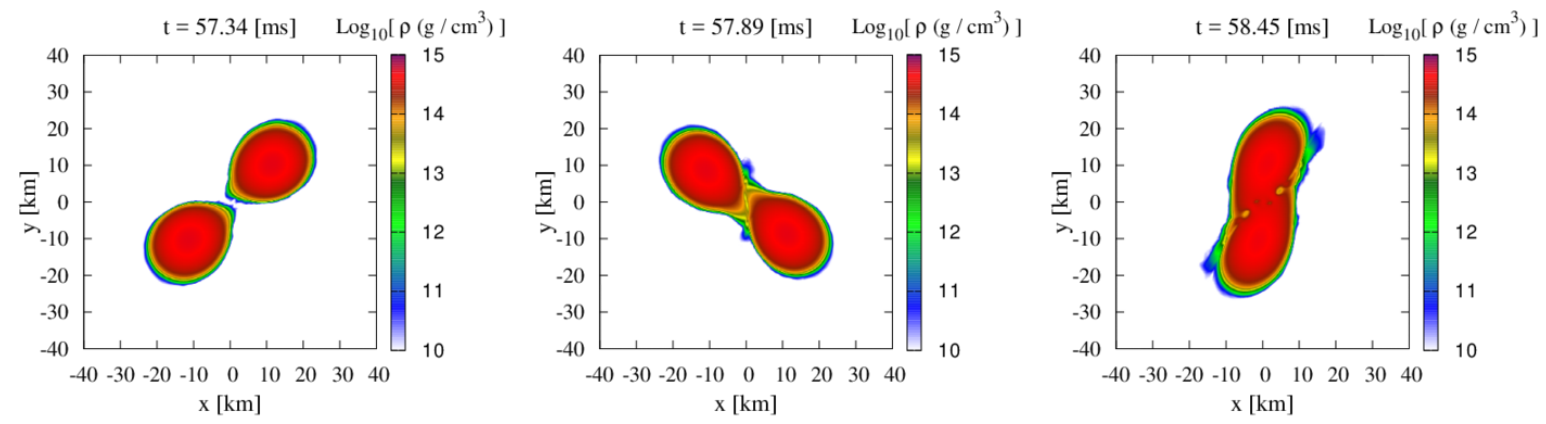

FIG. 7. Density profiles on the equatorial plane for the model $15 \mathrm{H} 135-135$ with $N=182$ at $t=t_{\text {peak }}-1.10 \mathrm{~ms}$ (left), $t_{\text {peak }}-0.55 \mathrm{~ms}$ (middle), and $t_{\text {peak }}$ (right).

these small-compactness models, two neutron stars come into contact at $t_{\text {cont }} \sim t_{\text {peak }}-1 \mathrm{~ms}$ (see the left panel of Fig. 7). Then, after the contact, the tidal deformation is further enhanced and a dumbbell-like object is formed (see the middle panel of Fig. 7). However, the density peaks of the dumbbell-like object are still clearly separated, and hence, the gravitational waveform has a chirp-type signal although the waveform is different slightly from the chirp signal from the separated body; that is, the evolution process of the system is determined by hydrodynamics equations (not simply by two-body equations of motion) and emission process of gravitational waves by the dumbbell-like object. As the distance between two density peaks decreases sufficiently, the gravitational-wave amplitude eventually reaches the peak (see the right panel of Fig. 7), and after the two density peaks merge, the amplitude significantly decreases. As mentioned above, for $t_{\text {cont }} \leq t_{\text {ret }} \leq t_{\text {peak }}$, chirp-type gravitational waves are emitted from a dumbbell-like object. However, the evolution of the object and the resulting gravitational waveforms cannot be well modeled by the current TEOB formalism because this stage is beyond the range of its application.

For the larger-compactness models, similar disagreement is found but only for the short duration because the tidal effects are weak, and furthermore, $t_{\text {peak }}-t_{\text {cont }}$ is $\lesssim 1 \mathrm{~ms}$ : For such compact models, the separation between two neutron stars is already small at $t_{\text {ret }}=t_{\text {cont }}$, and hence, the time scale of gravitational radiation reaction is as short as or shorter than the orbital period. Therefore, the duration of the stage of the dumbbelllike configuration is quite short and the disagreement between the NR and TEOB waveforms are not very remarkable.

Figure 5 and Table III show that irrespective of the numerical data employed, we obtain approximately the same results for the comparison between the NR and TEOB waveforms. This reconfirms that for $N \gtrsim 150$, an approximately convergent waveform (but with the phase error of order $0.1 \mathrm{rad}$ ) can be obtained in our numerical implementation.

To quantify the disagreement between the NR and TEOB waveforms, we define a measure of the mismatch by

$$
\mathcal{M}\left(t_{\mathrm{ret}}\right):=1-\frac{\left|\left(h_{\mathrm{NR}} \mid h_{\mathrm{TEOB}}\right)\right|}{\left(h_{\mathrm{TEOB}} \mid h_{\mathrm{TEOB}}\right)^{1 / 2}\left(h_{\mathrm{NR}} \mid h_{\mathrm{NR}}\right)^{1 / 2}}
$$

where $\left(h_{1} \mid h_{2}\right)$ is a function of $t_{\text {ret }}$ defined (without referring to detector noises) by

$$
\left(h_{1} \mid h_{2}\right):=\int_{t_{i}}^{t_{\mathrm{ret}}} h_{1}\left(t_{\mathrm{ret}}^{\prime}\right) h_{2}^{*}\left(t_{\mathrm{ret}}^{\prime}\right) d t_{\mathrm{ret}}^{\prime},
$$

and $h^{*}$ denotes the complex conjugate of $h$. Here, $t_{i}$ is chosen to be $20 \mathrm{~ms}$, and $h_{\mathrm{NR}}$ and $h_{\mathrm{TEOB}}$ denote the NR and TEOB waveforms, respectively.

Figure 8 plots $\mathcal{M}(t)$ for all the models with $N \geq 150$ that we consider in this paper. This shows that the degree of the mismatch steeply increases for the last inspiral orbits, in particular for the stiff EOS, due to the lack of modeling in the TEOB formalism as mentioned above. On the other hand, up to $\sim t_{\text {peak }}-3 \mathrm{~ms}$, the match is quite good: $\mathcal{M}$ is smaller than $10^{-3}$. This convinces us that the final issue in the TEOB formalism, in particular for the stiff EOS of large tidal deformability, is to take into account dynamics and the waveform in the dumbbell-like object phase. The phase difference, $\Phi_{\mathrm{NR}}-\Phi_{\mathrm{TEOB}}$, as well as the mismatch, $\mathcal{M}$, at a given moment of the retarded time increases nonlinearly with $\tilde{\Lambda}$. This suggests that for the improvement of the TEOB approach, a new nonlinear term of the tidal deformability is needed. This point will be discussed in our accompanying paper 59.

\section{SUMMARY}

We presented our latest numerical-relativity results of long-term, high-accuracy simulations for the inspiraling binary neutron stars. The simulations were performed 

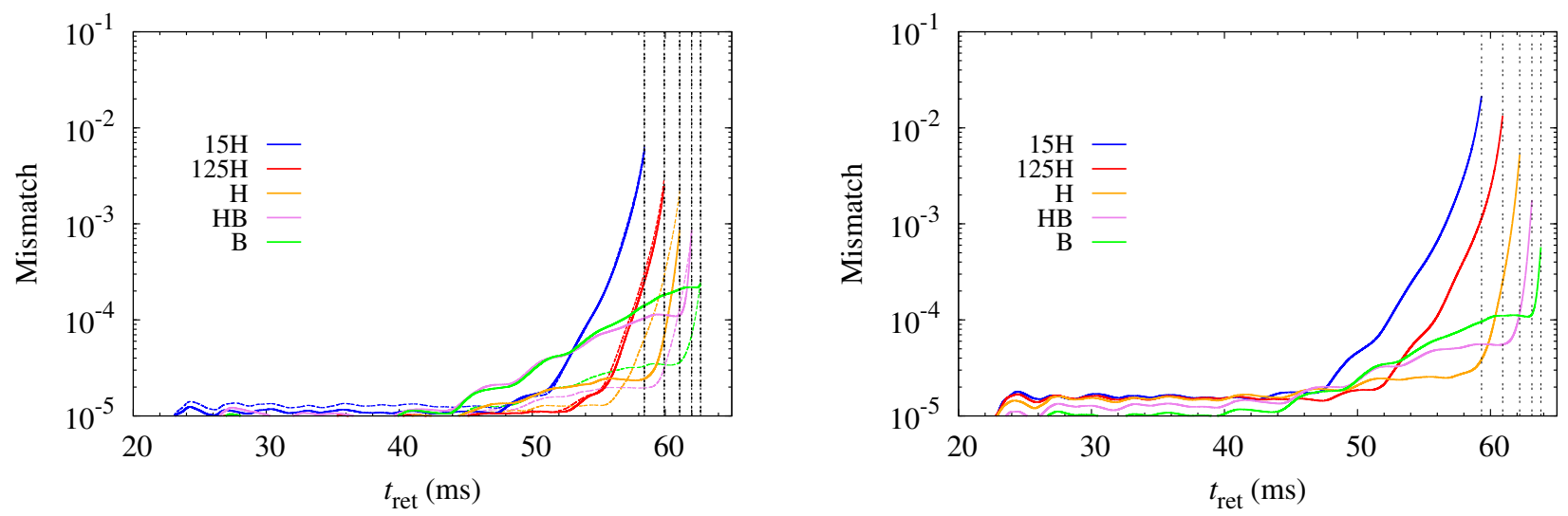

FIG. 8. $\mathcal{M}(t)$ for the equal-mass models (left panel) and for the unequal-mass models (right panel). In the left panel, the thick and thin curves denote the results for the numerical waveforms with $N=182$ and 150 , respectively. In the right panel, the thick curves denote the results for $N=150$. The vertical lines denote $t_{\text {peak }}$ for each model.

not only for the equal-mass binaries but also for the unequal-mass ones. We showed that if the grid resolution is high enough (i.e., the neutron-star radii are covered with the grid of its spacing $60-80 \mathrm{~m}$ ), it is possible to obtain a nearly convergent gravitational waveform (with the phase error of order $0.1 \mathrm{rad}$ ) from inspiraling binary neutron stars.

By comparing our high-resolution waveforms with the TEOB waveforms, we find that the TEOB formalism can reproduce accurate waveforms for binary neutron stars up to $\sim t_{\text {peak }}-3 \mathrm{~ms}$ irrespective of the neutron star EOS models. However, it is also found that for $t_{\text {peak }}-3 \mathrm{~ms} \lesssim$ $t \lesssim t_{\text {peak }}$ (in particular for $t_{\text {peak }}-1 \mathrm{~ms} \lesssim t \lesssim t_{\text {peak }}$ ), the current TEOB formalism cannot reproduce the numerical waveforms, in particular for the binary neutron stars of stiff EOS, and the phase error between the numerical and TEOB waveforms cannot be negligible as $\gtrsim 1 \mathrm{rad}$ at the amplitude peak for the stiff EOS models. The primary reason for this is that for such a stage, the evolution of the system cannot be well reproduced by the current TEOB equation of motion.

Accurate numerical data is crucial for modeling gravitational waveforms in the frequency domain [60]. Our numerical waveforms are the most accurate ones among those have been ever derived. We are now developing a phenomenological model from our numerical waveforms in the frequency domain, since we find that the numerical waveforms have a quality enough for the modeling. The results will be presented in our accompanying paper [59].

\section{ACKNOWLEDGMENTS}

We thank Tanja Hinderer, Ben Lackey, and Andrea Taracchini for helpful discussions and for telling us the details of the latest TEOB formalism. Numerical computation was performed on $\mathrm{K}$ computer at AICS (project numbers hp160211 and hp170230), on Cray
XC30 at cfca of National Astronomical Observatory of Japan, FX10 and Oakforest-PACS at Information Technology Center of the University of Tokyo, HOKUSAI FX100 at RIKEN, and on Cray XC40 at Yukawa Institute for Theoretical Physics, Kyoto University. This work was supported by Grant-in-Aid for Scientific Research $(24244028,16 \mathrm{H} 02183$, JP16H06342, JP17H01131, $15 \mathrm{~K} 05077,17 \mathrm{H} 06361)$ of JSPS and by a post-K computer project (Priority issue No. 9) of Japanese MEXT. Kawaguchi was supported by JSPS overseas research fellowships.

\section{Appendix A: The effects of residual eccentricity in the numerical waveforms}

We here demonstrate that the numerical waveforms in the early stage of numerical simulations are not suitable for the precise analysis because of the presence of the unphysical and irregular modulation in the angular frequency in the numerical waveforms, which is likely to be generated due to an unphysical setting of initial conditions (see below).

The modulation in the angular frequency of the numerical waveforms is extracted in the following procedure. First, we fit and subtract the non-oscillatory (i.e., physical) part of the numerical angular frequency by employing a function of the form

$$
\omega_{\text {Fit }}(t)=\sum_{n=0, n \neq 1}^{7} a_{n}\left(t_{1}-t\right)^{-(n+3) / 8} .
$$

The choice of this form is motivated by the Taylor-T3 approximant [6, 61], and the coefficients, $t_{1}$ and $a_{n}$, are determined by the least-square fitting procedure. The fitting is performed for $5 \mathrm{~ms} \leq t_{\text {ret }} \leq 50 \mathrm{~ms}$ of the waveforms. We checked that the result of the fitting depends only weakly on the choice of the time window. 

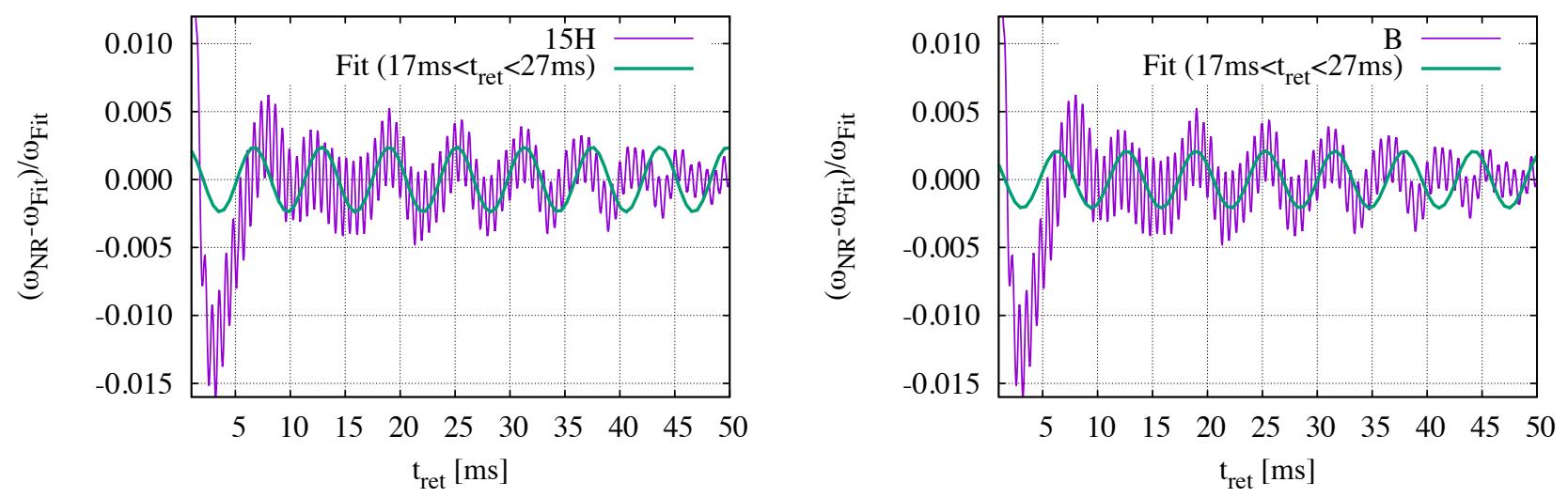

FIG. 9. The modulation in the angular frequency of the numerical waveforms for $15 \mathrm{H}$ (left panel) and B (right panel) EOS. The fitting curves are determined from the data in $17 \mathrm{~ms} \leq t_{\mathrm{ret}} \leq 27 \mathrm{~ms}$ by a sinusoidal function. For these fitting curves, the eccentricity of the binary orbits is measured to be $e \approx 1.19 \times 10^{-3}$ and $1.05 \times 10^{-3}$ for $15 \mathrm{H}$ and B EOS, respectively.

Figure 9 shows the modulation in the angular frequency of the numerical waveforms for the equal-mass model with $15 \mathrm{H}$ and B EOS with $N=150$. The modulation is defined by

$$
\frac{\omega_{\mathrm{NR}}-\omega_{\mathrm{Fit}}}{\omega_{\mathrm{Fit}}}
$$

where $\omega_{\mathrm{NR}}$ is the angular frequency of the numericalrelativity waveforms. The fitting curves in this figure are determined from the data in $17 \mathrm{~ms} \leq t_{\text {ret }} \leq 27 \mathrm{~ms}$ assuming that the modulation is written as a sinusoidal function. For $e \ll 1$, the residual eccentricity $e$ of the binary orbits is related to the amplitude of the modulation in the angular frequency, $\Delta \omega$, by $\Delta \omega \approx 2 e \omega$ assuming that the Newtonian relation is satisfied. For these fitting curves, the eccentricity of the binary orbits is measured to be $e \approx 1.19 \times 10^{-3}$ and $1.05 \times 10^{-3}$ for $15 \mathrm{H}$ and $\mathrm{B}$ EOS, respectively. Figure 9 shows that the eccentricity decreases with time, and it is $\lesssim 10^{-3}$ for $\gtrsim 20 \mathrm{~ms}$ irrespective of the EOS and binary mass employed.

Figure 9 shows that in the early part of the evolution with $t_{\text {ret }} \lesssim 15 \mathrm{~ms}$, the modulation in the angular frequency behaves in an irregular manner, although that for $t_{\text {ret }} \gtrsim 15 \mathrm{~ms}$ exhibits a simple damped-oscillationlike feature (neglecting its fine structure). That is, for $t_{\text {ret }} \lesssim 15 \mathrm{~ms}$, the center of the oscillation is not located at zero. This irregular oscillation causes an irregular error in the gravitational-wave phase and makes it difficult to perform a careful comparison between the numerical and TEOB waveforms. Therefore, we discard the waveforms in the early stage, and use only the data with $t_{\text {ret }} \geq 20 \mathrm{~ms}$ for the comparison in this paper. We note that even when we discard the data with $t_{\text {ret }} \leq 20 \mathrm{~ms}$, i.e., first $\sim 6.5-7$ wave cycles, we still have $\gtrsim 22$ cycles in the waveforms up to $t_{\text {peak }}$.

Our interpretation for this irregular modulation for the first $\sim 15 \mathrm{~ms}$ is that burst-like junk radiation is emitted just after the simulation is started, and it takes about
$15 \mathrm{~ms}$ until the system relaxes to a quasi-stationary state. The junk radiation is caused by unphysical setting of the initial condition associated with the so-called conformal flatness approximation for the initial-data problem (e.g., see chapter 5 of Ref. [62]).

\section{Appendix B: Phase error due to the finite-radius extraction and violation of the baryon mass conservation}

According to the Nakano's method Eq. 2.10), we extrapolate the gravitational waveforms extracted at $r_{0} / m_{0}=156,178$, and 200 to infinity. We find that the extrapolated waveforms slightly deviate each other due to the finite-radius extraction. By assuming this error falls off as $1 / r_{0}$, we obtain a waveform at infinity from the waveforms generated by the Nakano's method. Figure 10 plots the phase difference between the waveform generated by the Nakano's method and the waveform at infinity as a function of the retarded time. The phase error due to the finite-radius extraction is as small as $\sim 0.01 \mathrm{rad}$ up to the peak amplitude time and it decreases as a function of the extraction radius. We find a similar trend in the other equal-mass models and the unequal-mass models.

The baryon mass conservation is slightly violated at the merger because the conservative mesh refinement is not implemented in our code [63, 64]. Following Ref. [31, we estimate the phase error due to the violation of the baryon mass conservation by

$$
\delta \Phi_{\mathrm{b}}=\omega t_{\mathrm{ret}} \frac{\Delta M_{\mathrm{b}}}{M_{\mathrm{b}}},
$$

where $\omega$ is the angular frequency Eq. (2.13) and $M_{\mathrm{b}}$ is the baryon mass. $\Delta M_{\mathrm{b}}$ is the violation of the baryon mass conservation. Figure 11 plots the estimated phase error due to the violation of the baryon mass conservation for 

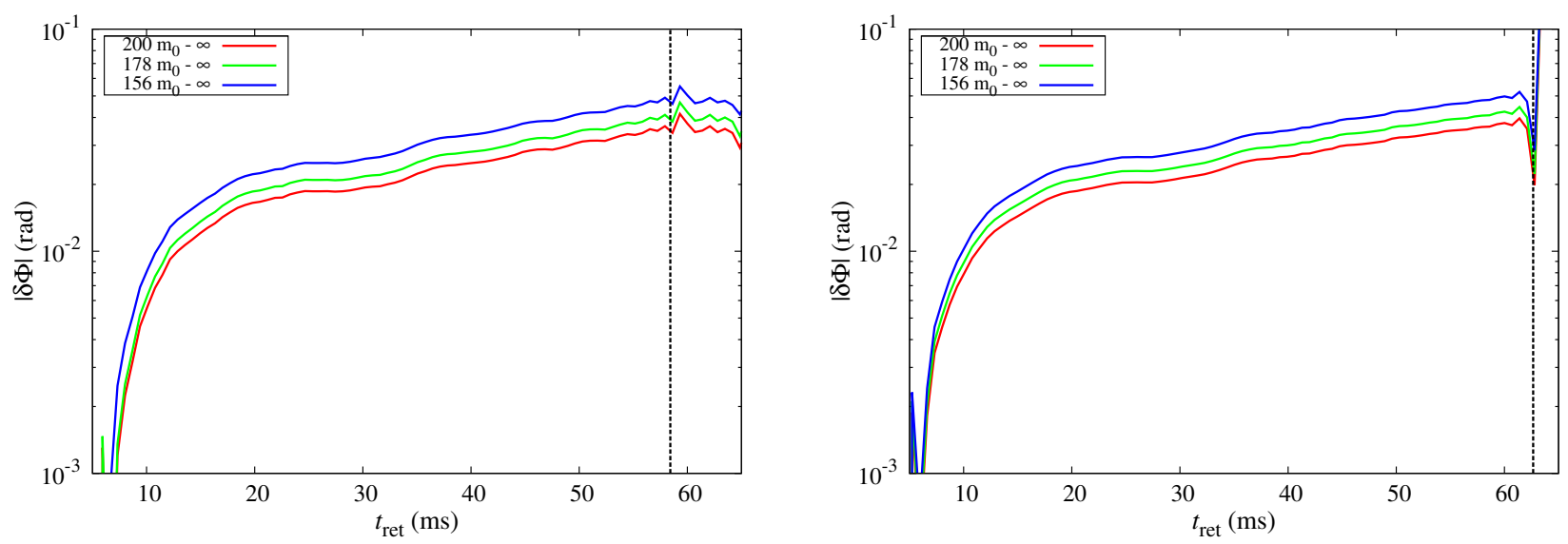

FIG. 10. Phase error due to the finite-radius extraction as a function of the retarded time for $15 \mathrm{H}$ EOS (left) and B EOS (right) for the equal-mass models with $N=182$. The vertical dotted lines are the peak amplitude time $t_{\text {peak }}$ (see the text in detail).

the equal-mass models with $N=182$. This plot shows that the resolution adopted in this work is high enough to reduce the error in the baryon mass conservation to $\approx 10^{-4} \%$ up to the peak amplitude time. Consequently, the phase error is $\approx O\left(10^{-4}\right)$ rad at the peak amplitude time irrespective of the models. The unequal-mass models show a similar result.
[1] J. Abadie et al. Nucl. Instrum. Meth. A 624, 223 (2010): Adcanced LIGO, http://www.advancedligo.mit.edu/ .

[2] B. P. Abbott et al., Phys. Rev. Lett. 116, 061102 (2016).

[3] B. P. Abbott et al., Phys. Rev. Lett. 116, 241103 (2016).

[4] B. P. Abbott et al., Phys. Rev. Lett. 117, 221101 (2017).

[5] B. P. Abbott et al., Phys. Rev. X 6, 041015 (2016).

[6] L. Blanchet. Gravitational Radiation from PostNewtonian Sources and Inspiralling Compact Binaries. Living Reviews in Relativity, 17:2, February 2014.

[7] B. P. Abbott et al., Phys. Rev. Lett. 116, 221101 (2016).

[8] T. Accadia et al. Class. Quant. Grav. 28, 025005 (2011) [Erratum-ibid. 28, 079501 (2011)]: Advanced VIRGO, http://www.cascina.virgo.infn.it/advirgo/

[9] K. Kuroda: LCGT collaboration, Class. Quantum Grav. 27, 084004 (2010).

[10] C. Cutler, and E. E. Flanagan, Phys. Rev. D 49, 2658 (1994).

[11] M. Hannam, D. A. Brown, S. Fairhurst, C. L. Fryer, and I. W. Harry, Astrophys. J. 766, 14 (2013).

[12] D. Lai, F. A. Rasio, and S. L. Shapiro, Astrophys. J. 420, 811 (1994).

[13] E. E. Flanagan, and T. Hinderer, Phys. Rev. D 77, 021502(R) (2008): J. Vines, T. Hinderer, and E. E. Flanagan, Phys. Rev. D 83, 084051 (2011).

[14] T. Hinderer, B. D. Lackey, R. N. Lang, and J. S. Read, Phys. Rev. D 81, 123016 (2010).

[15] D. Bini, T. Damour, and G. Faye, Phys. Rev. D 85, 124034 (2012).

[16] J. S. Read, L. Baiotti, J. D. E. Creighton, J. L. Friedman, B. Giacomazzo, K. Kyutoku, C. Markakis, L. Rezzolla, M. Shibata, and K. Taniguchi, Phys. Rev. D 88, 044042 (2013).

[17] L. Wade, J. D. E. Creighton, E. Ochsner, B. D. Lackey,
B. F. Farr, T. B. Littenberg, and V. Raymond, Phys, Rev. D 89, 103012 (2014).

[18] M. Agathos, J. Meidam, W. Del Pozzo, T. G. F. Li, M. Tompitak, J. Veitch, S. Vitale, C. Van Den Broeck, Phys. Rev. D 92, 023012 (2015).

[19] M. Favata, Phys. Rev. Lett. 112, 101101 (2014).

[20] K. Yagi and N. Yunes, Phys. Rev. D 89, 021303 (2014).

[21] K. Hotokezaka, K. Kyutoku, H. Okawa, and M. Shibata, Phys. Rev. D 91, 064060 (2015).

[22] A. Buonanno and T. Damour, Phys. Rev. D 59, 084006 (1999)

[23] B. D. Lackey and L. Wade, Phys. Rev. D 91, no. 4, 043002 (2015)

[24] D. Bini and T. Damour, Phys. Rev. D 90, 124037 (2014).

[25] S. Bernuzzi, A. Nagar, T. Dietrich, and T. Damour, Phys. Rev. Lett. 114, 161103 (2015).

[26] T. Hinderer et al., Phys. Rev. Lett. 116, 181101 (2016).

[27] J. Steinhoff, T. Hinderer, A. Buonanno and A. Taracchini, Phys. Rev. D 94, no. 10, 104028 (2016)

[28] T. Damour, A. Nagar, and L. Villain, Phys. Rev. D 85, 123007 (2012).

[29] K. Hotokezaka, K. Kyutoku, Y. Sekiguchi, and M. Shibata, Phys. Rev. D 93, 064082 (2016).

[30] In Ref. [21], the size of the finest AMR domains is about 1.15 times as large as the neutron star radii, i.e., $L / 2^{l}=$ $1.15 R_{\mathrm{NS}}$ with $l=8$. In this work, we conservatively set the finest AMR domain size as $1.35 R_{\mathrm{NS}}$.

[31] T. Dietrich and T. Hinderer, Phys. Rev. D 95, no. 12, 124006 (2017)

[32] T. Dietrich, S. Bernuzzi, and W. Tichy, arXiv:1706.02969

[33] S. Bernuzzi, T. Dietrich and A. Nagar, Phys. Rev. Lett. 115, no. 9, 091101 (2015) 


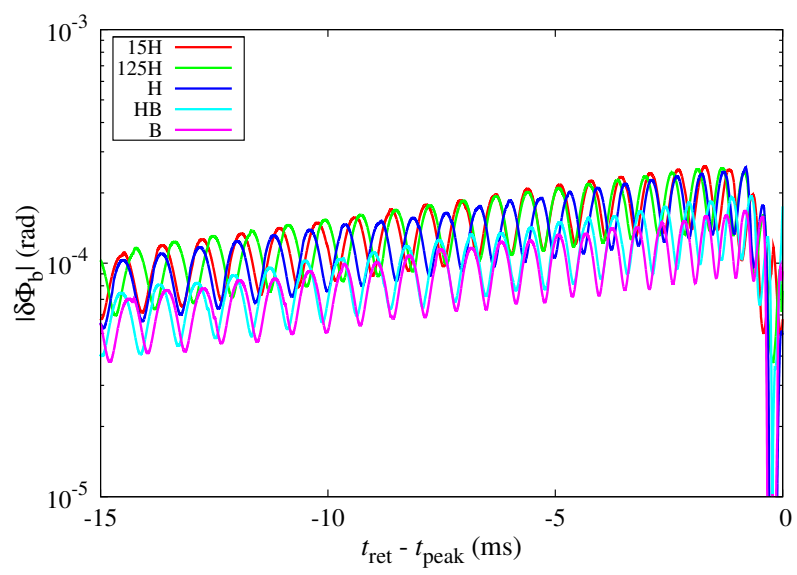

FIG. 11. Phase error due to the violation of the baryon mass conservation as a function of the retarded time for the equal-mass models with $N=182$. The vertical dotted lines show the peak amplitude time of each model.

[34] J. S. Read, C. Markakis, M. Shibata, K. Uryu, J. C. Creigton, and J. Friedman, Phys. Rev. D 79, 124033 (2009).

[35] L. Baiotti, T. Damour, B. Giacomazzo, A. Nagar, and L. Rezzolla, Phys. Rev. D 84, 024017 (2011).

[36] M. Thierfelder, S. Bernuzzi, and B. Brügmann, Phys. Rev. D 84,044012 (2011).

[37] S. Bernuzzi, M. Thierfelder, and B. Brügmann, Phys. Rev. D 85, 104030 (2012).

[38] S. Bernuzzi, A. Nagar, M. Thierfelder, and B. Brügmann, Phys. Rev. D 86, 044030 (2012).

[39] K. Hotokezaka, K. Kyutoku, and M. Shibata, Phys. Rev. D 87, 044001 (2013).

[40] D. Radice, L. Rezzolla, and F. Galeazzi, Mon. Not. Roy. Soc. 437, L46 (2014).

[41] R. Haas et al., Phys. Rev. D 93, 124062 (2016).

[42] P. C. Peters, Phys. Rev. 136, B1224 (1964).

[43] T. Yamamoto, M. Shibata, and K. Taniguchi, Phys. Rev. D 78, 064054 (2008).

[44] M. Shibata and T. Nakamura, Phys. Rev. D 52, 5428(1995): T. W. Baumgarte and S. L. Shapiro, Phys. Rev. D 59, 024007(1998): M. Campanelli, C. O. Lousto, P. Marronetti, and Y. Zlochower, Phys. Rev. Lett. 96, 111101 (2006): J. G. Baker, J. Centrella, D.-I. Choi, M. Koppitz, and J. van Meter, Phys. Rev. Lett. 96, 111102 (2006).

[45] D. Hilditch, S. Bernuzzi, M. Thierfelder, Z. Cao, W. Tichy, and B. Brügmann, Phys. Rev. D 88, 084057 (2013).

[46] K. Kyutoku, M. Shibata, and K. Taniguchi, Phys. Rev. D 90, 064006 (2014).

[47] LORENE webpage: http://www.lorene.obspm.fr/ .

[48] L. Bildsten, and C. Cutler, Astrophys. J. 400, 175 (1992).

[49] C. S. Kochanek, Astrophys. J. 398, 234 (1992).
[50] J. S. Read, B .D. Lackey, B. J. Owen, and J. L. Friedman, Phys. Rev. D 79, 124032 (2009).

[51] B. D. Lackey, K. Kyutoku, M. Shibata, P. R. Brady, and J.L. Friedman, Phys. Rev. D 85, 044061 (2012).

[52] P. B. Demorest, T. Pennucci, S. M. Ransom, M. S. E. Roberts, and J. W. T. Hessels, Nature 467, 1081 (2010): J. Antoniadis et al., Science, 340, 448 (2013).

[53] C. O. Lousto, H. Nakano, Y. Zlochower, and M. Campanelli, Phys. Rev. D 82, 104057 (2010).

[54] H. Nakano, Class. Quantum Grav. 32, 177002 (2015).

[55] H. Nakano, J. Healy, C. O. Lousto and Y. Zlochower, Phys. Rev. D 91, no. 10, 104022 (2015)

[56] C. Reisswig and D. Pollney, Class. Quantum Grav. 28, 195015 (2011).

[57] K. Kawaguchi, K. Kyutoku, H. Nakano, and M. Shibata, arXiv: 1705.07459

[58] L. Rezzolla and K. Takami, Phys. Rev. D 93, 124051 (2016).

[59] K. Kawaguchi, et al., in preparation.

[60] S. Khan, S. Husa, M. Hannam, F. Ohme, M. Prrer, X. Jimnez Forteza, and A. Boh, Phys. Rev. D 93, 044007 (2016).

[61] A. Buonanno, B. R. Iyer, E. Ochsner, Y. Pan, and B. S. Sathyaprakash. Comparison of postNewtonian templates for compact binary inspiral signals in gravitational-wave detectors. Phys. Rev. D, 80(8):084043, October 2009.

[62] M. Shibata, Numerical Relativity (World Scientific, 2016).

[63] W. E. East, F. Pretorius and B. C. Stephens, Phys. Rev. D 85, 124010 (2012) doi:10.1103/PhysRevD.85.124010

[64] T. Dietrich, S. Bernuzzi, M. Ujevic and B. Brgmann, Phys. Rev. D 91, no. 12, 124041 (2015) doi:10.1103/PhysRevD.91.124041 